\title{
CD45RO and CD45RA positive cell populations in idiopathic membranous and IgA glomerulopathy
}

\author{
A H S Lee, P S Bass, J H Williams, B Evans, D B Jones, J M Theaker, D R Davies
}

\begin{abstract}
Aims-To immunophenotype and quantitate glomerular and interstitial inflammatory cells in cases of idiopathic membranous and IgA glomerulopathy; to correlate cell numbers with aspects of clinical data and renal function.

Methods-Routine indirect immunoperoxidase staining was performed on frozen section renal biopsy specimens for $T$ and $B$ lymphocyte related antigens, macrophages and MHC class II antigens. Double immunohistochemical staining was performed to identify CD45RO + and CD45RA + cells.
\end{abstract}

Results-In IgA glomerulopathy correlations were found relating interstitial cell numbers to creatinine concentration at biopsy (CD45RO + and CD45RA + cells) and follow up creatinine concentration $(\mathrm{CD} 3+, \mathrm{CD} 4+, \mathrm{CD} 8+$, CD45RO +, and CD45RA + cells). Also in IgA glomerulopathy mean arterial pressure at biopsy correlated with interstitial cell numbers and most recent follow up creatinine concentration. There were no correlations between glomerular inflammatory cells and renal function in either disease. Double staining showed that although most glomerular CD45RO + and CD45RA + cells were macrophages, positive cells in the interstitium were lymphocytes. The interstitial CD45RO+: RA + ratio in normal renal biopsy specimens was approximately 5:1; for IgA glomerulopathy it was 1.5 and was 1.0 in idiopathic membranous glomerulopathy. Conclusions-This study demonstrates that interstitial, and not glomerular, inflammatory cell numbers correlate with renal function in primary glomerular disease and that double staining is necessary to interpret positive immunostaining for antigens located on more than one type of inflammatory cell. Detailed investigation of the interstitial CD45RO + and CD45RA + cells may give an insight into the pathogenesis of glomerular disease. (f Clin Pathol 1996;49:43-47)

Keywords: IgA glomerulopathy, idiopathic membranous glomerulopathy, immunophenotype, glomerular cells, interstitial cells.

Most forms of primary glomerulopathy involve acute or chronic immunological damage to the glomerulus. ${ }^{1}$ However, studies have shown that, for many types of glomerular disease, it is the extent of the tubulo-interstitial damage that dictates the degree of impairment of renal function and survival. It has been suggested that tubular damage, possibly mediated by glomerular leakage of tubulopathic proteins or the production of paracrine factors by glomerular cells, may trigger the production of cytokines or activation of complement components by tubules. These cytokines and complement components recruit inflammatory cells into the interstitium and stimulate fibroblasts (and possibly tubular cells themselves) to produce collagen, leading to scarring (reviewed in ${ }^{2-4}$ ).

CD45 (leucocyte common antigen, LCA) is a family of glycoproteins found on the surface of all nucleated haematopoietic cells. ${ }^{5}$ Several isoforms of the CD45 protein exist, including CD45RA and CD45RO, and these molecules are involved in various aspects of lymphocyte activation. Studies have shown that CD45RA is expressed on naive (unprimed) T cells, whilst $T$ cells that have encountered antigen are $\mathrm{CD} 45 \mathrm{RO}+$. The number of CD45RO + cells increases with age until, in adulthood, approximately $45 \%$ of the $T$ cell population expresses this antigen. Most $\mathrm{T}$ cells in normal and inflamed tissues are $\mathrm{CD} 45 \mathrm{RO}+{ }^{6}$

We postulate that the renal tubulo-interstitium is an area of active cell mediated immunity in primary glomerular disease and that recruited interstitial $T$ cells and macrophages play a central role in the evolution of irreversible damage to this compartment.

To examine this hypothesis we used renal biopsy specimens from patients with idiopathic membranous or IgA glomerulopathy (Berger's disease, IgA glomerulopathy) to immunophenotype glomerular and interstitial inflammatory cells and, using double staining, identify and quantify the CD45RO + and CD45RA + cells; and to correlate the cell types with aspects of clinical history and renal function.

\section{Methods}

Biopsy specimens from 18 patients with idiopathic membranous and 20 with IgA glomerulopathy were studied. Three normal biopsy specimens (by light and electron microscopy and immunohistology) were used as controls. Two of the control biopsy specimens were from patients with unexplained haematuria, one from a patient with loin pain/haematuria.

The following clinical details were recorded: age, sex, mode of presentation, and duration 
Panel of primary antibodies

\begin{tabular}{llll}
\hline$C D$ & Antibody & Source & Specificity of antibody \\
\hline CD3 & UCHT1 & P C L Beverley, ICRF & Pan T cell marker \\
CD4 & 73F11 & Tenovus, Southampton & T helper \\
CD8 & OKT8 & Ortho Diagnostics & T suppressor \\
CD45RO & UCHL1 & P C L Beverley, ICRF & Helper/inducer (primed) subset of CD4 \\
CD45RA & WR16 & Tenovus, Southampton & Suppressor (naive) subset of CD4 \\
CD37 & WR17 & Tenovus, Southampton & Pan B cell \\
CD11c & $3 \cdot 9$ & N Hogg, ICRF & Macrophages \\
- & WR18 & Tenovus, Southampton & MHC class II \\
- & CAM5·2 & Becton Dickinson & Cytokeratin \\
\hline
\end{tabular}

of symptoms to renal biopsy. In addition, mean arterial pressure (diastolic blood pressure plus one third of the pulse pressure), serum creatinine concentration, proteinuria (g/day), and glomerular filtration rate (GFR) at the time of biopsy were recorded. Post-biopsy details of serum creatinine concentration (and GFR and treatment when known) were also recorded. Formal statistical analysis was not performed for GFR or treatment, as details of these were available in less than half the patients.

\section{IMMUNOHISTOCHEMISTRY}

Sections, $4 \mu \mathrm{m}$ thick, were cut from frozen renal biopsy specimens. The sections were stained with a panel of antibodies (table), using an indirect immunoperoxidase method. ${ }^{7}$ Briefly, the sections were fixed in water free acetone at room temperature for 15 minutes. The primary antibodies, at appropriate dilutions, were applied for 30 minutes and then rabbit anti-mouse immunoglobulin peroxidase conjugate (diluted in TBS) applied for a further 30 minutes. Diaminobenzidine solution was applied for 10 minutes. Haematoxylin was used as the counterstain. Tonsillar tissue and renal biopsy specimens with a notable interstitial infiltrate (for example, near end stage kidney) were used as positive controls. The primary antibody was omitted for negative controls.

\section{DOUBLE IMMUNOHISTOCHEMISTRY}

Six biopsy specimens each of idiopathic membranous and IgA glomerulopathy were stained by double immunohistochemistry. Sections were cut, fixed and primary antibodies used as described earlier. The rabbit anti-mouse immunoglobulin peroxidase conjugate solution in TBS was then used at twice the normal concentration. This was applied for 30 minutes, followed by 3-amino-9-ethylcarbazole (AEC) substrate. The following were then applied: the second primary antibody (for 30 minutes); goat anti-mouse immunoglobulin (30 minutes); and mouse anti-alkaline phosphatase complex in TBS (30 minutes). Fast Blue substrate was then added for about five minutes. Sections were mounted using an aqueous mountant.

The following pairs of antibodies were used: UCHT1/UCHL1, UCHT1/WR16, WR16/ UCHL1, UCHL1/3.9, and WR16/3.9 (first antibody visualised via AEC, second by Fast Blue; as a check this was then reversed. In addition, each primary antibody was also visualised by AEC alone and Fast Blue alone.)

\section{QUANTITATION}

All cell counts were performed without reference to clinical details. Only cells with an identifiable nucleus and membrane staining were regarded as positive. The area of the biopsy specimen was measured using Improvision image analysis. The tubulo-interstitial area was then calculated by subtracting glomeruli and larger calibre vessels (that is, small and medium sized arteries and veins). In addition, the number of patent and segmentally and globally sclerosed glomeruli was noted. Cell counts in patent glomeruli and the tubulointerstitium were then expressed per $\mathrm{mm}^{2}$ (and number/patent glomeruli). For WR18 only, 10 high power fields were used to count interstitial cells using a graticule (E35, Graticules, Tonbridge, Kent, UK; area $=10 \times 0.0657 \mathrm{~mm}^{2}$ ). In the double immunohistochemistry slides formal counting was performed only for glomerular cells.

\section{STATISTICS}

Spearman's rank correlation $\left(\mathrm{r}_{\mathrm{S}}\right)$ was used to analyse the data. In all cases, only $p$ values equal to, or less than, 0.02 were regarded as significant.

\section{Results}

\section{CLINICAL DETAILS}

Two of the three patients in the control group were 29 years of age and the other was 50 years old. Two were male. All three had a normal serum creatinine concentration and urinary protein excretion.

There were 16 men and four women in the IgA glomerulopathy group (age range 14-74 years, median 33 years) and 13 men and five women in the idiopathic membranous glomerulopathy group (age range 22-78 years, median 43 years; two men in this group had two biopsy specimens taken). Median length of follow up for the IgA glomerulopathy group was 21 months and for the idiopathic membranous glomerulopathy group was $\mathbf{3 0}$ months. In the idiopathic membranous glomerulopathy group only, serum creatinine concentrations at biopsy correlated with the degree of proteinuria $\left(r_{S}=0.64, p=0.02\right)$, the duration of disease $\left(r_{s}=0.65, p=0.01\right)$ and mean arterial pressure $\left(r_{s}=0.72, p=0.009\right)$. In the IgA glomerulopathy group, the mean arterial pressure correlated with most recent follow up serum creatinine concentration $\left(r_{s}=0.87, p=0.001\right)$. 
CELL POPULATIONS

Normal biopsy specimens

The mean number of $\mathrm{CD} 3+, \mathrm{CD} 8+$ and $\mathrm{CD} 45 \mathrm{RO}+$ glomerular cells was in the range $4-15 / \mathrm{mm}^{2}$. There were larger numbers of CD11c + and CD45RA + cells (31 and 48 cells $/ \mathrm{mm}^{2}$, respectively). There were no glomerular CD4 + or CD37 + cells. There was uniform, global glomerular positivity for WR18 because of endothelial cell staining and thus quantitation of WR18+ cells was not attempted.

There were very few interstitial $\mathrm{CD} 37+$, CD45RA +, CD11c +, or CD4 + cells (mean 10 cells or less $\left./ \mathrm{mm}^{2}\right)$. CD $45 \mathrm{RO}+\left(36 / \mathrm{mm}^{2}\right)$ and CD3 + cells $\left(52 / \mathrm{mm}^{2}\right)$ were more numerous. There were very large numbers of WR $18+$ cells $\left(214 / \mathrm{mm}^{2}\right.$, mainly endothelial cells, some inflammatory cells).

\section{Idiopathic glomerulopathies}

The following findings were common to IgA and idiopathic membranous glomerulopathy. Glomeruli-The mean number of $\mathrm{CD} 3+$, CD4 + and CD8 + cells was in the range 5-27/ $\mathrm{mm}^{2}$. There were larger (mean) numbers of glomerular CD45RO $+\left(29 / \mathrm{mm}^{2}\right.$ in idiopathic membranous glomerulopathy and $39 / \mathrm{mm}^{2}$ in IgA glomerulopathy), CD45RA + (52 and 63, respectively) and CD11c + cells (60 and 90, respectively). There was a strong correlation between the number of glomerular CD4 + and $\mathrm{CD} 8+$ cells $\left(\mathrm{r}_{\mathrm{s}}\right.$ for idiopathic membranous glomerulopathy $=0.60$ and for $\operatorname{IgA}$ glomerulopathy $=0.65 ; \mathrm{p}=0.001$ and 0.006 ) and a moderate correlation between the numbers of CD4 + and CD45RA + cells $\left(r_{s}=0.53\right.$ and $0.51, p=0.015$ and 0.01 ). There was also a strong correlation between the number of glomerular CD45RO+ and CD45RA + cells $\left(r_{S}=0.68\right.$ and $0.53, p=0.005$ and 0.008$)$. There were virtually no glomerular CD37+ cells $\left(0.8 / \mathrm{mm}^{2}\right.$ in idiopathic membranous glomerulopathy; none in IgA glomerulopathy).

There was no correlation between any of the glomerular cell populations and renal function or other clinical data. No correlations were found between glomerular and interstitial cell populations.

Tubulo-interstitium - There were few interstitial CD37 + cells (mean $12 / \mathrm{mm}^{2}$ for idiopathic membranous glomerulopathy and $7 \cdot 3 / \mathrm{mm}^{2}$ for IgA glomerulopathy). Otherwise there were very large numbers of positive cells in this compartment $(\mathrm{CD} 3+=204$ and 179 , respectively; approximate range for CD4 + and CD8 + cells $=50-75$ and for CD45RA + and $\mathrm{RO}+$ cells $=75-125$ ). The mean number of WR18 + cells was increased (257 and 305, respectively). Except for $\mathrm{CD} 37+$ and $\mathrm{CD} 11 \mathrm{c}+$ cells in idiopathic membranous glomerulopathy $\left(r_{s}=0.45, p=0.022\right)$, there was a moderate to strong correlation between all types of interstitial inflammatory cells $\left(r_{S}=\right.$ $0.51-0.93, \mathrm{p}=0.02-<0.0000001$ )

The following differences were noted between IgA and idiopathic membranous glomerulopathy.
IgA glomerulopathy only-There was a strong correlation between the serum creatinine concentration at biopsy and all types of interstitial cells, except WR18 + $\left(\mathrm{r}_{\mathrm{S}}=0.53-0.69, \mathrm{p}=\right.$ $0.002-0.0003)$. The correlation between interstitial CD45RO + and RA + cells and biopsy serum creatinine concentration was particularly strong $\left(r_{s}=0.67, p=0.0006\right.$ and $r_{s}=0.69, p=$ 0.0003 , respectively). In addition, there was a strong correlation between the interstitial $\mathrm{CD} 3+, \mathrm{CD} 4+$ and CD8 + cells $\left(\mathrm{r}_{\mathrm{s}}=0.69\right.$ $0.83, p=0.002-0.00004)$ and a moderate correlation between interstitial CD45RA + and $\mathrm{CD} 45 \mathrm{RO}+\left(\mathrm{r}_{\mathrm{s}}=0.62, \mathrm{p}=0.007 ; \mathrm{r}_{\mathrm{s}}=0.54, \mathrm{p}=\right.$ 0.02 ) and the most recent (follow up) creatinine concentration. Mean arterial pressure (at biopsy) correlated with interstitial $\mathrm{CD} 3+$, $\mathrm{CD} 4+$ and CD8 + $\left(\mathrm{r}_{\mathrm{s}}=0.62-0.77, \mathrm{p}=0.016\right.$ 0.002 ).

Idiopathic membranous glomerulopathy onlyThere was a correlation between the glomerular and interstitial CD45RO + to RA + cell ratio $\left(r_{S}=0.71, p=0.0003\right)$, and two patients with deteriorating renal function who each had two biopsy specimens taken showed a noticeable increase in interstitial cell numbers in the second biopsy specimen, associated with the functional deterioration.

\section{DOUBLE IMMUNOHISTOCHEMISTRY}

The results of double immunohistochemical staining were similar for both glomerulopathies. Thus, up to $80 \%$ of the glomerular CD45RA + cells and a smaller proportion of CD45RO + cells were also CD11c +, indicating these cells were macrophages rather than lymphocytes. This finding was confirmed by the quantitative results of the single immunohistochemistry study (cell counts shown in the figure, panel A). In contrast, the total number of interstitial $\mathrm{CD} 45 \mathrm{RO}+$ and CD45RA + cells (sum of means approximately 200 cells $/ \mathrm{mm}^{2}$ for both glomerulopathies) was comparable with the numbers of $\mathrm{CD} 3+$ and CD4 + cells (approximately $250 / \mathrm{mm}^{2}$ ), but much greater than the number of CD11c + cells $\left(36 / \mathrm{mm}^{2}\right.$ for IgA glomerulopathy and $65 / \mathrm{mm}^{2}$ for idiopathic membranous glomerulopathy), suggesting that the vast majority of interstitial CD45RA + and RO + cells were $\mathrm{T}$ lymphocytes (figure, panel $\mathrm{B}$ ).

\section{Discussion}

There are a number of clinicopathological factors which, at the time of biopsy, are known to indicate adverse renal survival in primary glomerular disease. Clinically, the presence of impaired renal function, proteinuria and hypertension correlate with poor outcome in many glomerulopathies, as does the extent of tubulo-interstitial damage and inflammation within the biopsy specimen. Several studies have shown a correlation between the interstitial inflammatory cell population ( $\mathrm{T}$ lymphocytes and macrophages) and the degree of renal impairment. ${ }^{8-10}$ However, to our knowledge, there is no information to date relating to the presence of CD45RA + and RO + cell populations in glomerulonephritis. We were 

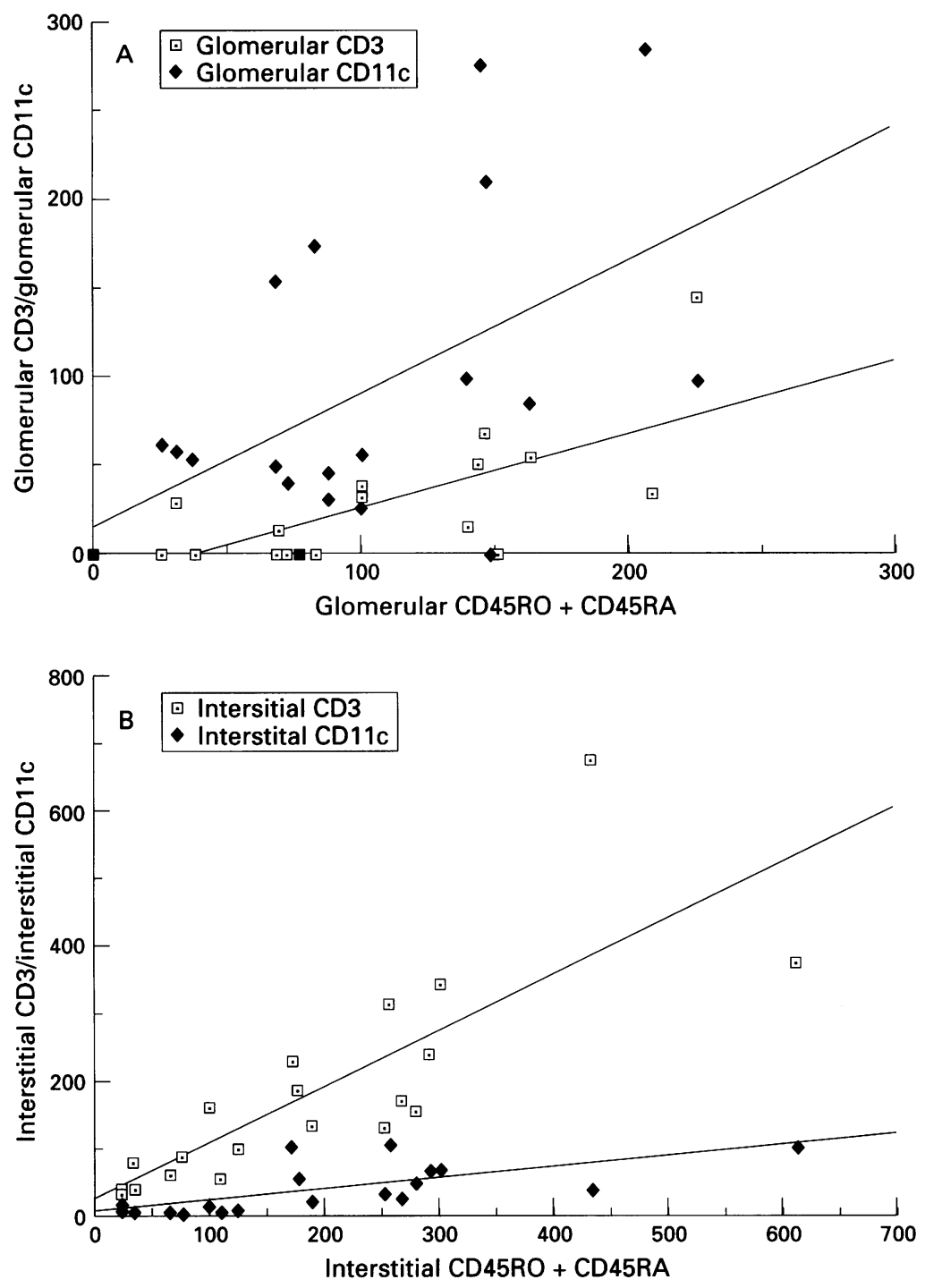

In the glomeruli $(A)$ of biopsy specimens showing $\operatorname{Ig} A$ nephropathy the number of $C D 45 R O$ and CD45RA cells is comparable with the number of CD11c cells, and larger than the number of CD3 cells. By contrast in the interstitium (B) the number of $C D 45 R O$ and $C D 45 R A$ cells is comparable with the number of CD3 cells and is larger than the number of CD11c cells. This is consistent with CD45RO and CD45RA predominantly staining macrophages in the glomeruli and $T$ cells in the interstitium.
Although the numbers of patients in the control and disease groups are relatively small, our results highlight some interesting similarities and important differences between the two glomerulopathies. Thus, as in the normal biopsy specimens, there were very few glomerular B lymphocytes and relatively small numbers of $\mathrm{T}$ cells. The mean number of glomerular CD45RO + and RA + cells far exceeded that of $\mathrm{CD} 3+(\mathrm{T})$ cells and approximated to the number of glomerular macrophages $(\mathrm{CD} 11 \mathrm{c}+)$. Double staining showed that the large majority of both the RA + and $\mathrm{RO}+$ cells were also CD11c +, indicating these cells were not, in fact, $T$ cells but macrophages. In common with several other studies we found no correlation between any of the glomerular inflammatory cell counts and aspects of renal function or other clinical data.

There were contrasting results relating to the importance of the interstitial cell populations in the two glomerulopathies. Although there were strong correlations between all the interstitial populations in both diseases, only in IgA glomerulopathy was there a significant correlation between the number of interstitial inflammatory cells and biopsy creatinine concentration. This correlation was particularly strong for the interstitial CD45RO + and RA + cell populations $\left(r_{s}=0.67-0.69, p=0.0003\right.$ $0.0006)$. For idiopathic membranous glomerulopathy, the correlation between interstitial CD45RO + cells or CD45RA + cells and biopsy creatinine concentration just failed to reach significance $\left(r_{S}=0.41\right.$ and $0 \cdot 40$, respectively, $p=0.05$ for both). The IgA glomerulopathy group also showed a correlation between the number of interstitial $\mathrm{CD} 3+$, $\mathrm{CD} 4+, \mathrm{CD} 8+$, and $\mathrm{CD} 45 \mathrm{RO}+$ and $\mathrm{RA}+$ cells and the follow up (latest) plasma creatinine concentration. In addition, in IgA glomerulopathy, there was good correlation between mean arterial pressure at biopsy and interstitial $\mathrm{CD} 3+, \mathrm{CD} 4+$ and $\mathrm{CD} 8+$ cell counts $\left(r_{s}=0.69-0.83, p=0.002-0.00007\right)$. Interestingly, mean arterial pressure also correlated strongly with latest (follow up) creatinine concentration $\left(r_{s}=0.87, p=0.001\right)$ and weakly with the creatinine concentration at biopsy $\left(r_{s}=0.42, p=0.06\right)$. Thus, there seems to be a relation among mean arterial pressure at biopsy, the number of interstitial $T$ cells and serum creatinine concentration (particularly follow up creatinine concentration). These findings do not indicate whether the $T$ cell infiltrate is a primary or secondary phenomenon, but emphasise the potentially important role of interstitial cells in renal function in IgA glomerulopathy.

Unlike the glomerular cell populations, double staining and cell counts showed that CD45RA + and RO + interstitial cells were lymphocytes rather than macrophages. $\mathrm{Al}$ though we have no information on the blood CD45RA + and RO + cell populations in our patients, it is interesting to compare the ratios of these cells within the renal interstitial tissue. The interstitial CD45RO + :RA + ratio in the normal biopsy specimens was about 5 to 1 whereas for IgA glomerulopathy it was 1.5 to 1 CD45RA and RO isoforms are not $T$ cell restricted and may be found on monocytes. ${ }^{11}$ We used double staining in order to determine the precise nature of the cells expressing CD45RA or RO in the biopsy tissue. 
and in idiopathic membranous glomerulopathy the ratio was about 1 to 1 . This apparent increment in the number of CD45RA + cells in the diseased biopsy specimens contrasts with reports in the literature for other inflammatory and auto-immune processes and suggests recruitment of naive lymphocytes into the interstitium. It may be that in both glomerular diseases, tubulopathic proteins that have passed through the glomerulus or paracrine factors produced by glomerular cells may cause tubular epithelial cell injury resulting in them presenting novel antigen(s) or producing chemoattractant cytokines. ${ }^{2}$ This could lead to the recruitment and expansion of interstitial $T$ cells (particularly CD45RA + cells) and macrophages. Ultimately, interstitial fibrosis could occur by cytokine/growth factor driven macrophage stimulation of fibroblasts.

The importance of the interstitial CD45RA + cells is reinforced by the observation that for IgA glomerulopathy (but not idiopathic membranous glomerulopathy), the number of interstitial CD45RA + and RO + cells correlates with creatinine concentration at biopsy and most recent follow up (the correlation for CD45RA + cells was stronger). However, interstitial cell populations (including CD45RA + and RO + cell numbers) did not correlate with biopsy or follow up creatinine concentrations in idiopathic membranous glomerulopathy. Although this may simply reflect the relatively small numbers in our patient group, an alternative explanation is based on the possible differences in the pathogenesis of idiopathic membranous glomerulopathy compared with IgA glomerulopathy. The immune complexes in idiopathic membranous glomerulopathy occur almost exclusively within the subepithelial aspect of the glomerular basement membrane and the glomerular cells stimulated and profile of growth factors/cytokines produced may lead to differ- ent events in the tubulo-interstitial compartment compared with the predominantly mesangial targeted immune deposits of IgA glomerulopathy.

In conclusion, our study adds to the body of evidence that tubulo-interstitial cell mediated events, and not those occurring in the glomerulus, correlate with renal function in primary glomerular disease. It also illustrates the problems associated with interpretation of positive immunostaining for cell markers such as CD45RA and CD45RO which are known to be present on more than one inflammatory cell type. In this type of case, double immunocytochemistry is essential.

This study was supported by the Wessex Regional Health Authority and the Wessex Medical School Trust.

1 Porter KA. Primary glomerulonephritis I. In: Porter KA Pugh RCB, Ansell ID, eds. The kidneys and the urinary tract. Vol 8 in the series Systematic pathology. Edinburgh: Churchill Livingstone, 1992:125-34.

2 Ong ACM, Fine LG. Tubular-derived cytokines and human renal disease progression. Nephrol Dial Transplant 1994;9: $471-2$.

3 Nath KA. Tubulointerstitial changes as a major determinant in the progression of renal damage. Am $\mathcal{F}$ Kidney Dis 1992; 20:1-17.

4 D'Amico G. Influence of clinical and histological features on actuarial renal survival in adult patients with idiopathic IgA nephropathy, membranous nephropathy, and memIgA nephropathy, membranous nephropathy, and mem-
branoproliferative glomerulonephritis: survey of the recent branoproliferative glomerulonephritis: survey

5 Thomas ML. The leukocyte common antigen family. $A n n$ Rev Immunol 1989;7:339-69.

6 Clement LT. Isoforms of the CD45 common leukocyte antigen family: markers for human T-cell differentiation. f Clin Immunol 1992;12:1-10.

7 Mepham BL, Britten KJM. Immunostaining methods for frozen and paraffin sections. In: Jones DB, Wright DH, eds. Lymphoproliferative diseases. Vol 15 in the series Immunology and medicine. Dordrecht: Kluwer Academic Publishers, 1990:187-211.

8 Hooke DH, Gee DC, Atkins RC. Leukocyte analysis using monoclonal antibodies in human glomerulonephritis. Kidney Int 1987;31:964-72.

9 Main IW, Nikolic-Paterson DJ, Atkins RC. T cells and macrophages and their role in renal injury. Semin Nephrol 1992;12:395-407.

10 Alexopoulos E, Seron D, Hartley RB, Nolasco F, Cameron JS. The role of interstitial infiltrates in IgA nephropathy: a study with monoclonal antibodies. Nephrol Dial Transplant 1989;4:187-95.

11 Lai R, Visser L, Poppema S. Tissue distribution of restricted leukocyte common antigens. A comprehensive study with protein- and carbohydrate-specific CD45R antibodies. Lab Invest 1991;684:844-54. 